\title{
Relationship between the Concentration of Anti-polyethylene Glycol (PEG) Immunoglobulin M (IgM) and the Intensity of the Accelerated Blood Clearance (ABC) Phenomenon against PEGylated Liposomes in Mice
}

\author{
Yosuke Hashimoto, ${ }^{a}$ Taro Shimizu, ${ }^{a}$ Amr Selim Abu Lila,${ }^{a, b}$ Tatsuhiro Ishida, ${ }^{*, a}$ and \\ Hiroshi Kiwada ${ }^{a}$ \\ ${ }^{a}$ Department of Pharmacokinetics and Biopharmaceutics, Subdivision of Biopharmaceutical Sciences, Institute of \\ Health Biosciences, The University of Tokushima; 1-78-1 Sho-machi, Tokushima 770-8505, Japan: and ${ }^{b}$ Department \\ of Pharmaceutics and Industrial Pharmacy, Faculty of Pharmacy, Zagazig University; Zagazig 44 519, Egypt. \\ Received September 11, 2014; accepted December 8, 2014
}

PEGylation, which is the surface modification of nanocarriers with polyethylene glycol (PEG), has increased the circulation time and reduced the immunogenic responses to nanocarriers. However, many reports have demonstrated that the intravenous injection of sterically stabilized PEGylated liposome (SL) causes an accelerated blood clearance (ABC) of subsequent doses via anti-PEG immunoglobulin M (IgM)-mediated complement activation. In the present study, the relationships between serum anti-PEG IgM concentration, the intensity of complement activation and the hepatic clearance of SL were quantitatively investigated for their role in the $A B C$ phenomenon. Interestingly, with increasing serum anti-PEG IgM concentrations, the intensity of complement activation increased linearly, while the intensity of the hepatic clearance of SL was increased and then saturated. In addition, only 15-17\% of anti-PEG IgM in blood circulation induced by SL at different doses was associated with a second dose SL. The present results indicate that it is the hepatic uptake of SL that is the limiting step in the $\mathrm{ABC}$ phenomenon, rather than the association of anti-PEG IgM to the $\mathrm{SL}$ and a subsequent complement activation.

Key words anti-polyethylene glycol (PEG); immunoglobulin M (IgM); PEGylated liposome (SL); accelerated blood clearance $(\mathrm{ABC})$ phenomenon; complement activation

Polyethylene glycol (PEG) is a water-soluble, bio-inert and non-toxic polymer that is widely used as an important excipient in pharmaceutical formulations. ${ }^{1,2)}$ PEGylation is the covalent attachment of PEG to a therapeutic agent/nanoparticle, and it has been extensively acknowledged for improved the pharmacokinetics by reducing the immunogenicity of pharmaceutical formulations. ${ }^{3-5)}$ PEG grafted at the surface of liposomes is thought to attract a water shell to the liposomal surface, thereby inhibiting the adsorption of opsonins, such as complements, and/or recognition by the host immune system, and, consequently, imparting long-circulating characteristics to liposomes. ${ }^{6,7)}$ These long-circulating characteristics of sterically stabilized PEGylated liposomes (SL), in addition to their small size $(<200 \mathrm{~nm})$, enable the efficient accumulation of PEGylated liposomes into solid tumors via an enhanced permeability and retention (EPR) effect. ${ }^{8)}$ This has led to the clinical approval of PEGylated liposome-based anti-tumor drug formulations. A typical example is doxorubicin-encapsulated PEGylated liposomes (Doxil ${ }^{\mathbb{R}}$ ), which has strong anti-tumor activity against a wide range of tumors. ${ }^{9)}$

However, we and others have reported that repeated injection of PEGylated nanoparticles, such as SL, induces an anti-PEG immunoglobulin $\mathrm{M}$ (IgM) response, which triggers the rapid hepatic uptake of subsequent doses. ${ }^{10)}$ This disappearance of subsequent doses via the production of anti-PEG $\mathrm{IgM}$ is referred to as the "accelerated blood clearance (ABC) phenomenon."10-13) The ABC phenomenon has been identified as comprising two phases: the induction phase, in which the biological system is "primed" by the first administration of SL, leading to the induction of an anti-PEG IgM response against the SL, and the effectuation phase that follows the second, or subsequent, administration in which the SL are rapidly opsonized and cleared from the blood circulation by Kupffer cells via complement receptor-mediated endocytosis/ phagocytosis $^{14)}$ via anti-PEG IgM-mediated activation of the complement system. ${ }^{10)}$ IgM has great potential to activate the complement system via classic pathway.

In the effectuation phase, there was a strong inverse relationship between the dose of initially injected SL and the extent of the ABC phenomenon. ${ }^{15}$ ) The ABC phenomenon was potentially manifested when lower phospholipid doses (0.001-0.1 $\mu \mathrm{mol}$ phospholipids $/ \mathrm{kg}$ ) of SL were intravenously injected as the first dose. On the other hand, higher phospholipid doses ( $\geq 5 \mu \mathrm{mol}$ phospholipids $/ \mathrm{kg}$ ) substantially abrogated the induction of the $\mathrm{ABC}$ phenomenon. It is assumed that at a low dose of phospholipids, the extent of B-cell receptor cross-linking by low dose SL might be sufficient to activate the cells and promote the production of specific antibody against PEG, i.e., anti-PEG IgM. On the other hand, higher doses of SL ( $\geq 5 \mu \mathrm{mol} / \mathrm{kg}$ ) might cause MZ-B cells to induce an immune tolerance or to be anergic; unable to mount a complete response against SL. In addition, in the effectuation phase, it is easy to be assumed that the dose of the second, or subsequent, administration, strongly affects the intensity of the ABC against the dose as a result of interaction with antiPEG IgM and subsequent complement activation-mediated endocytosis/phagocytosis.

In earlier studies, we showed that the intensity of the $A B C$ 
of subsequent doses of SL are correlated with the concentration of anti-PEG IgM in serum. ${ }^{10)}$ However, the rate-limiting steps of the ABC phenomenon remain unclear-the source of the complement system or the capacity of the hepatic uptake of a second dose of SL. In the present study, therefore, we investigated the quantitative correlation between the serum concentration of anti-PEG IgM which induced by changing the first dose of SL, the complement activation against a second dose of SL, and the intensity of the hepatic uptake of a second dose of SL.

\section{MATERIALS AND METHODS}

Materials Hydrogenated egg phosphatidylcholine (HEPC) and 1,2-distearoyl-sn-glycero-3-phosphoethanolamine- $n$-[methoxy (PEG)-2000] ( $\mathrm{mPEG}_{2000}$-DSPE) were generously donated by NOF (Tokyo, Japan). Cholesterol (CHOL) was purchased from Wako Pure Chemical Industries, Ltd. (Osaka, Japan). ${ }^{3} \mathrm{H}$-Cholesterylhexadecyl ether $\left({ }^{3} \mathrm{H}-\mathrm{CHE}\right)$ was purchased from PerkinElmer, Inc., Japan (Yokohama, Japan). All lipids were used without further purification. All other reagents were of analytical grade.

Animals Male BALB/c mice weighting 20-22g were purchased from Japan SLC (Shizuoka, Japan). The mice had free access to water and mouse chow, and were housed under controlled environmental conditions (constant temperature, humidity and a $12 \mathrm{~h}$ dark/light cycle). All animal experiments were evaluated and approved by the Animal and Ethics Review Committee of the University of Tokushima.

Preparation of SL SL, composed of HEPC:CHOL: $\mathrm{mPEG}_{2000}$-DSPE (1.85:1.0:0.15 $\mathrm{M}$ ratio), was prepared as previously described. ${ }^{15}$ ) The mean diameter of the resultant liposome was $105.0 \pm 4.3 \mathrm{~nm}$, as determined using a NICOMP 370 HPL submicron particle analyzer (Particle Sizing System, CA, U.S.A.). The concentration of phospholipid (PL) was determined via a colorimetric assay. ${ }^{16)}$ To follow the biodistribution of the liposome, it was labeled with a trace amount of ${ }^{3} \mathrm{H}-\mathrm{CHE}$ $(40 \mu \mathrm{Ci} / \mu \mathrm{mol} \mathrm{PL})$ as a non-exchangeable lipid-phase marker.

Determination of Anti-PEG IgM Concentration in Serum At day 5 following the injection of different doses of SL $(0.01,0.1$ or $5 \mathrm{nmol} \mathrm{PL} / \mathrm{mouse}$ ) into mice, a blood sample was withdrawn from the tail veins, and a serum was prepared. Anti-PEG IgM levels in the serum samples were detected using a simple enzyme-linked immunosorbent assay (ELISA), as described previously. ${ }^{17)}$ When determining the concentration of anti-PEG IgM in the serum, an anti-PEG IgM monoclonal antibody, HIK-M09, that we had recently generated ${ }^{18)}$ was used as a standard. The ELISA system showed a good linear relationship between the absorption and the anti-PEG IgM concentration (see supplemental Fig. 1), as well as good reproducibility.

In Vivo Complement Activation Study To investigate the in vivo complement activation following the injection of a second dose of SL, mice were intravenously injected with a second test dose $(100 \mathrm{nmol} \mathrm{PL} /$ mouse) on day 5 following the first injection with different doses $(0.01,0.1$ or $0.5 \mathrm{nmol}$ $\mathrm{PL} /$ mouse). Naïve mice served as a control. Two minutes following injection of the second dose, blood was withdrawn $(400 \mu \mathrm{L})$ from the inferior vena cava into a microtube. The tube was pretreated with $400 \mu \mathrm{L}$ of $0.1 \mathrm{M}$ ethylenediaminetetraacetic acid (EDTA) ( $\mathrm{pH}$ 7.4) to stop further complement

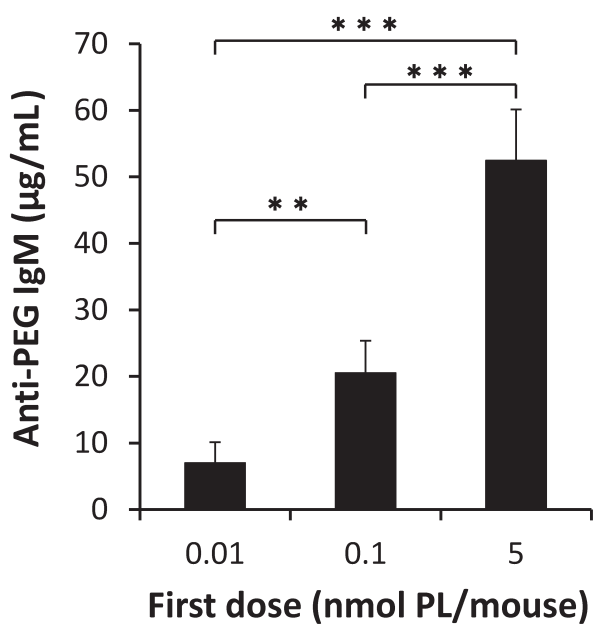

Fig. 1. Serum Anti-PEG IgM Concentration

At day 5 following the intravenous injection of different doses of SL $(0.01,0.1$ or $5 \mathrm{nmol} \mathrm{PL} / \mathrm{mouse}$ ), blood was collected from the tail vein. Serum anti-PEG IgM was determined. Data represent the mean \pm S.D. $(n=3)$. ${ }^{* *} p<0.01, * * * p<0.005$

activation. Then, the tube was centrifuged at $1000 \mathrm{rpm}$ and $4^{\circ} \mathrm{C}$ for $10 \mathrm{~min}$ to separate the plasma sample. Ten micro-liters of plasma samples (1:100 dilution) were separated on a $12.5 \%$ sodium dodecyl sulfate-polyacrylamide gel electrophoresis (SDS-PAGE) gel (e • PAGEL, ATTO, Tokyo, Japan) under reducing conditions, and were then transferred electrophoretically onto a Hybond-ECL (GE Healthcare, Chalfont, U.K.). The membrane was blocked with phosphate-buffered saline (PBS) containing $0.05 \%$ Tween-20 and $1 \%$ nonfat dry milk powder ( $\mathrm{pH}$ 7.4) for $1 \mathrm{~h}$ at room temperature. After three washes with PBS containing $0.05 \%$ Tween-20 ( $\mathrm{pH} 7.4$ ), membranes were incubated with horseradish peroxidase (HRP) conjugated goat anti-mouse C3 (Valeant Pharmaceuticals International, CA, U.S.A.) (1:1000) for $1 \mathrm{~h}$ at room temperature. After an additional three washes with PBS containing $0.05 \%$ Tween-20 $(\mathrm{pH}$ 7.4), the membranes were processed for enhanced chemiluminescence using ECL Plus Chemiluminescence Reagent (GE Healthcare), images were obtained using a LAS-4000 EPUV mini, and were analyzed via a Multi Gauge v.3.2 (FUJIFILM, Tokyo, Japan).

Biodistribution and Pharmacokinetic Study Pharmacokinetic parameters are used as an indicator of the intensity of the ABC phenomenon of PEGylated nanoparticles. ${ }^{13,19)}$ To elucidate the intensity of the ABC phenomenon of SL following the injection of a second dose, the pharmacokinetic parameters from 0 to $60 \mathrm{~min}$ post-injection were used. Mice were intravenously injected with radio-labeled test doses (100 nmol PL/mouse) on day 5 after the first injection $(0.01$, 0.1 or $0.5 \mathrm{nmol} \mathrm{PL} / \mathrm{mouse})$. Non-pretreated naïve mice served as a control. At different time points (2, 15, 30 and $60 \mathrm{~min})$ after the test dose injection, samples (blood $[100 \mu \mathrm{L}]$, liver and spleen) were collected. Tissue samples were washed and weighed after removal of the excess fluid. The radioactivity in the samples was assayed, as described previously. ${ }^{20)}$ To calculate the pharmacokinetic parameters (the area under the blood concentration-time curve $(A U C)$, hepatic clearance $(C L h)$ and splenic clearance $(C L s)$ ) from the non-linear pharmacokinetics, a bootstrap method ${ }^{21)}$ was employed as follows.

Step 1: One sample was randomly chosen from out of 3 samples at each timepoint, which allowed the construction of 
pseudo time-course profiles.

Step 2: $A U C, C L h$ and $C L s$ (statistics; $X$ ) were calculated from each time course, as constructed in Step 1.

Step 3: Steps 1 and 2 were repeated for $B$ (bootstrap number) times. The mean $(\bar{X})$ and the S.D. of $A U C, C L h$ and $C L s$ were calculated as follows:

$$
\bar{X} \frac{1}{B} \sum_{(i=1)}^{n} X i \text {, S.D. }(\bar{X})=\sqrt{\frac{1}{B-1}} \sum_{i=1}^{B}(X i-\bar{X})^{2}
$$

where the number of resampling $B$ was set at 1000 in the present study. The $A U C$ was calculated by the area under the blood concentration-time curve from 0 to $60 \mathrm{~min}$ post-injection, and the CLh and CLs were calculated as follows:

$$
C L h=X h_{(60 \mathrm{~min})} / A U C_{(0 \rightarrow 60 \mathrm{~min})}, \quad C L s=X s_{(60 \mathrm{~min})} / A U C_{(0 \rightarrow 60 \mathrm{~min})}
$$

where $X h_{(60 \mathrm{~min})}$ and $X s_{(60 \mathrm{~min})}$ were the amount of SL accumulated in the liver and spleen, respectively, at $60 \mathrm{~min}$ postinjection.

Number of Anti-PEG IgM per One SL Particle To evaluate the number of anti-PEG IgM per SL in the blood circulation, SL was isolated from the blood circulation and the amount of associated anti-PEG IgM on SL was measured. Briefly, mice were intravenously injected with a second test dose (100 nmol PL/mouse) on day 5 following the first injection with different doses $(0.01,0.1$ or $0.5 \mathrm{nmol} \mathrm{PL} / \mathrm{mouse})$. Naïve mice served as a control. Two minutes after the second dose injection, blood was withdrawn from the inferior vena cava and plasma was prepared, as described above. To isolate the SL from the plasma, gel filtration chromatography was employed, as described earlier. ${ }^{22}$ Forty-five micro-liters of isolated SL $(0.6 \mathrm{~mm})$ were lysed by the addition of $5 \mu \mathrm{L}$ of $20 \%$ CHAPS solution and anti-PEG IgM concentration in a 160 -fold diluted lysed liposome solution that was measured as described above.

The particle number of SL $\left(N_{\mathrm{SL}}\right)$ was calculated as follows:

$$
N_{\mathrm{SL}}=N_{\mathrm{a}} /\left[\pi / a_{\mathrm{L}}\left\{R^{2}+(R-2 t)^{2}\right\}\right]
$$

where $N_{\mathrm{a}}$ is the Avogadro constant, $t$ is the bilayer thickness, $R$ is the diameter of $\mathrm{SL}$, and $a_{\mathrm{L}}$ is the average headgroup area per lipid. The bilayer thickness was assumed to be $40 \AA$ and $a_{\mathrm{L}}$ was calculated using values of 71,41 and $19 \AA^{2}$ for phosphatidylcholine, phosphatidylethanolamine, and $\mathrm{CHOL}$, respectively, as weighted by the appropriate mole fraction of each component. ${ }^{23)}$ The value of $a_{\mathrm{L}}$ for SL was calculated as $52.2 \AA^{2} /$ lipid.

The number of anti-PEG IgM was calculated as follows:

$$
N_{\mathrm{IgM}}=N_{\mathrm{a}} \cdot C_{\mathrm{L}} / M
$$

where $C_{\mathrm{L}}$ is the amount of anti-PEG $\operatorname{IgM}$ in lysed SL solution and $M$ is the molecular mass of $\operatorname{IgM}$. The molecular mass of $\mathrm{IgM}$ was assumed to be $900000 \mathrm{~g} / \mathrm{mol}$.

The number of anti-PEG IgM per one SL particle $\left(N_{\mathrm{IgM} / \mathrm{SL}}\right)$ was calculated as follows:

$$
N_{\mathrm{IgM} / \mathrm{SL}}=N_{\mathrm{IgM}} / N_{\mathrm{SL}}
$$

The assumed maximum number of associated anti-PEG
IgM per one SL particle $\left(N_{\mathrm{IgM} / \mathrm{SL}(\max )}\right)$ was calculated as follows:

$$
N_{\mathrm{IgM} / \mathrm{SL}(\max )}=N_{\mathrm{a}} \cdot C S \cdot V S /\left(M \cdot D \cdot N_{\mathrm{SL}}\right)
$$

where $C s$ is the serum anti-PEG IgM concentration, $V_{S}$ is the assumed serum volume and $D$ is the dose of SL. The serum volume of a Balb/c mouse was assumed to be $0.87 \mathrm{~mL}$.

Statistics All values are expressed as the mean \pm S.D. Statistical analysis was performed with a two-tailed unpaired $t$ test using GraphPad InStat software (GraphPad Software, CA, U.S.A.). The level of significance was set at $p<0.05$.

\section{RESULTS}

Effect of the First SL Dose on the Induction of AntiPEG IgM Anti-PEG IgM concentration was assessed on day 5 after a single SL injection with different doses $(0.01,0.1$ and $5 \mathrm{nmol} \mathrm{PL} / \mathrm{mouse})$. As shown in Fig. 1, within the tested dose range, serum anti-PEG IgM concentrations increased proportionally with an increase in the injected dose. An injection of $\mathrm{SL}$ at a dose of $5 \mathrm{nmol} \mathrm{PL} / \mathrm{mouse}$ induced the maximum level of anti-PEG $\operatorname{IgM}(52.5 \mu \mathrm{g} / \mathrm{mL})$ and showed a 7.5 - and 2.9fold increase in the anti-PEG IgM concentration compared with an injection of SL at 0.01 and $0.1 \mathrm{nmol} \mathrm{PL} /$ mouse, respectively.

Complement Activation by a Second Dose Many research groups have reported that the complement system is activated by the anti-PEG IgM and consequently SL is opsonized. This indicates that the intensity of complement activation may be an indicator of the intensity of the ABC phenomenon. However, the relationship between the concentration of serum anti-PEG IgM and the intensity of complement activation under the $\mathrm{ABC}$ phenomenon remains unclear.

$\mathrm{C} 3$, which plays a pivotal role in opsonizing invasive pathogens and foreign nanoparticles, ${ }^{24)}$ consists of two chains $(\alpha$ and $\beta$ ). During complement activation, the $\mathrm{C} 3 \alpha$ chain is cleaved and $\alpha 1$ and $\alpha 2$ fragments are generated. The most prominent and easily detectable fragment is $\alpha 2$, which is frequently used as an indicator of the intensity of complement activation. ${ }^{25)}$ Therefore, we decided to measure the amount of generated $\mathrm{C} 3 \alpha 2$ fragment in blood circulation following the injection of a second dose of SL ( $100 \mathrm{nmol} \mathrm{PL} / \mathrm{mouse})$ into the mice pretreated with a first dose of SL $(0.01,0.1$ or $5 \mathrm{nmol} \mathrm{PL/}$ mouse).

As shown in Fig. 2A, the $\alpha 2$ fragment in the plasma obtained from the pretreated mice increased with an increase in the dose of pretreated SL, while no $\alpha 2$ fragment was induced in the non-treated (naïve) mice. To elucidate the relationship between the intensity of complement activation and serum anti-PEG IgM concentration, bands of $\alpha 2$ fragment in Fig. 2A were quantified using a computing densitometer (Fig. 2B). It is interesting that there was a good linear correlation between the quantity of generated $\mathrm{C} 3 \alpha 2$ and the concentration of the anti-PEG IgM in the serum. This result indicates that in vivo complement activation under the $\mathrm{ABC}$ phenomenon was induced in an anti-PEG IgM concentration-dependent manner.

Biodistribution and Pharmacokinetics of a Second Dose of SL Pharmacokinetic parameters have been used as an indicator of the intensity of the ABC phenomenon. ${ }^{10)}$ To study 


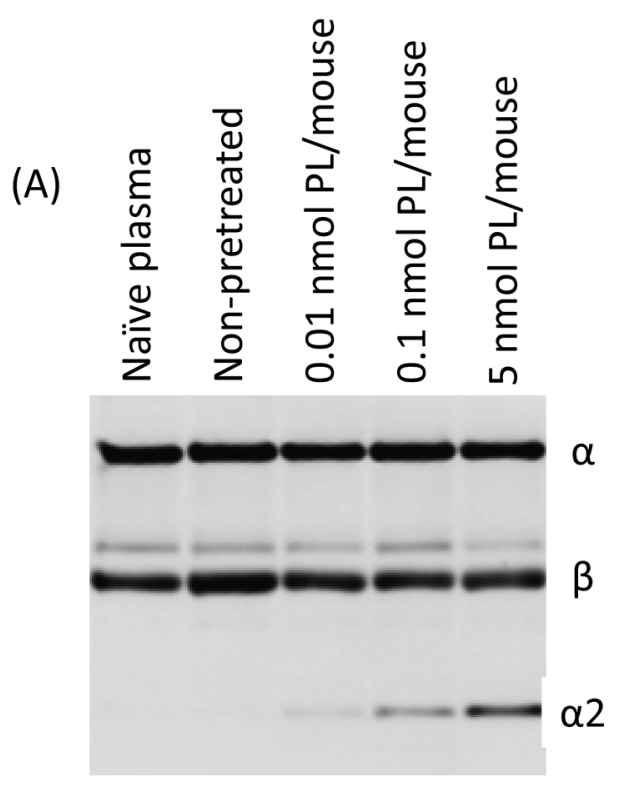

(B)

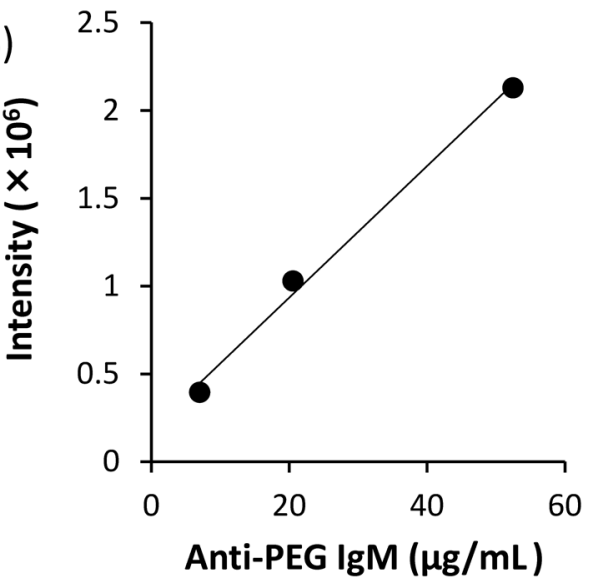

Fig. 2. Effect of Serum Anti-PEG IgM Concentration on the Intensity of Complement Activation

Mice were intravenously injected with SL $(0.01,0.1$ or $5 \mathrm{nmol} \mathrm{PL} / \mathrm{mouse}) 5 \mathrm{~d}$ prior to an intravenous second injection of SL ( $200 \mathrm{nmol} \mathrm{PL} /$ mouse). (A) Complement activation was detected by visualizing the bands for the $\mathrm{C} 3$ cleavage product, $\alpha 2$ fragments, in plasma by Western blotting. (B) Quantitative evaluation of the levels of $\alpha 2$ fragment in plasma.

the relationship between the concentration of anti-PEG $\operatorname{IgM}$ and the intensity of the ABC phenomenon against SL (a rapid clearance and enhanced uptake by liver), the biodistribution of test SL was studied under the ABC phenomenon. A second test dose of radio-labeled SL ( $100 \mathrm{nmol} \mathrm{PL} / \mathrm{mouse})$ was given on day 5 after the first injection of SL with $0.01,0.1$ or $5 \mathrm{nmol}$ $\mathrm{PL} / \mathrm{mouse}$. Consistent with our earlier study, ${ }^{10,22)}$ the pretreatment with SL resulted in an enhanced blood clearance (Fig. $3 \mathrm{~A}$ ), and the preferential liver accumulation (Fig. 3B) of the test second dose in a dose-dependent manner. The rank order of the disappearance of SL from blood circulation and the increased hepatic uptake of SL was $5>0.1>0.01 \mathrm{nmol}$ PL of $\mathrm{SL}$ received by mice as a first dose. This order correlated with the level of serum anti-PEG IgM. Consistent with our earlier study, ${ }^{26)}$ no changes in the pattern of splenic accumulation
(A)

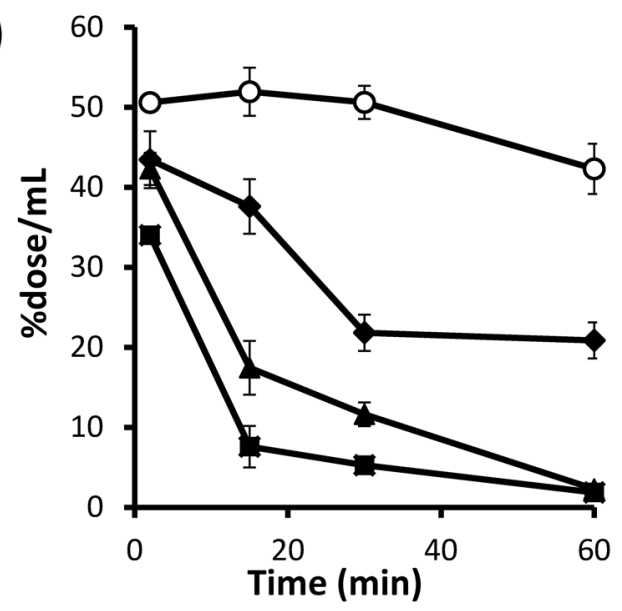

(B)

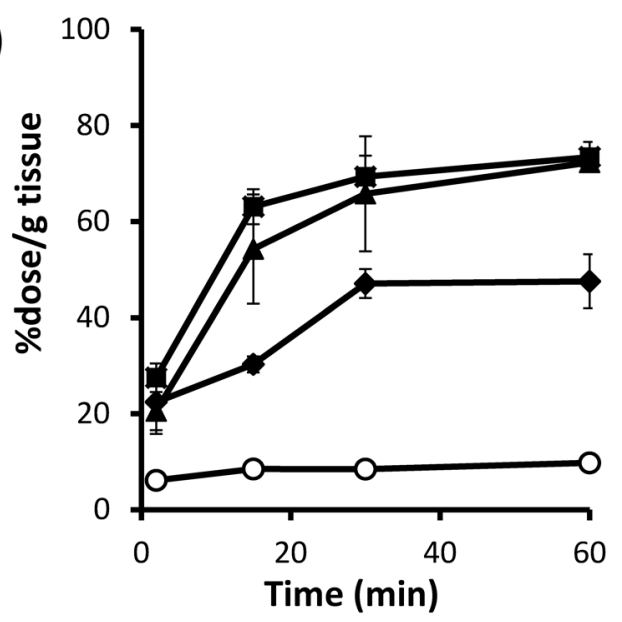

(C)

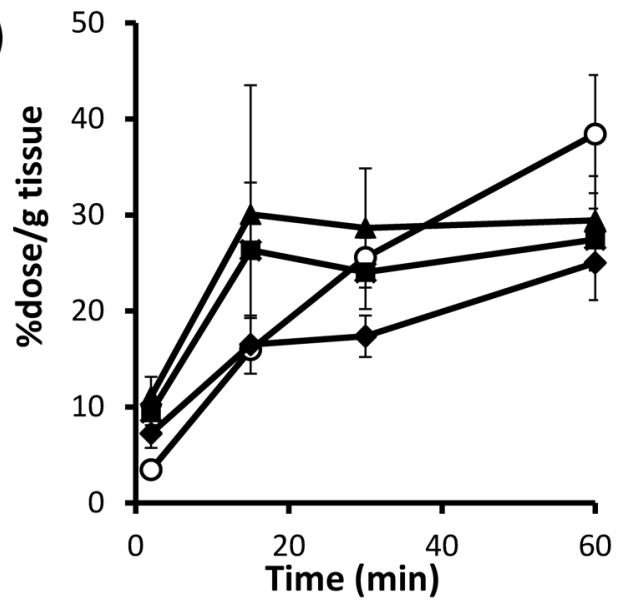

Fig. 3. Pharmacokinetics and Organ Distribution of a Second Dose of SL

At day 5 after pretreatment with SL $[0.01(\diamond), 0.1(\mathbf{\Delta})$ or $5(\boldsymbol{\square}) \mathrm{nmol} \mathrm{PL} / \mathrm{mouse}]$ ${ }^{3} \mathrm{H}$-CHE-labeled second test doses of SL $(100 \mathrm{nmol} \mathrm{PL} / \mathrm{mouse})$ were intravenously injected. Non-pretreated naïve mice $(O)$ were used as a control. At different timepoints post-injection $(2,15,30$ and $60 \mathrm{~min})$, radio-labeled SL in blood (A), liver (B) and spleen $(\mathrm{C})$ was evaluated. Data represent the mean \pm S.D. $(n=3)$.

profiles were observed in all treated groups (Fig. 3C).

The pharmacokinetic parameters of test-SL are summarized in Table 1. Normally, SL showed a relatively larger AUC $(2.94 \%$ dose/mL $/ \mathrm{min})$ and lower CLh and CLs (3.60 and $1.28 \mathrm{~mL} / \mathrm{min}$, respectively). In the $\mathrm{ABC}$ phenomenon, however, the value of $A U C$ was decreased, but that of the $C L h$ and 
Table 1. Pharmacokinetic Parameters of a Second Dose of SL

\begin{tabular}{cccc}
\hline \hline First dose $(\mathrm{nmol} \mathrm{PL} / \mathrm{mouse})$ & $A U C\left(\times 10^{-3}\right)(\%$ dose $/ \mathrm{mL} \cdot \mathrm{min})$ & $C L h\left(\times 10^{-3}\right)(\mathrm{mL} / \mathrm{min})$ & $C L s\left(\times 10^{-3}\right)(\mathrm{mL} / \mathrm{min})$ \\
\hline Non-pretreated & $2.94 \pm 0.63$ & $3.60 \pm 0.46$ & $1.28 \pm 0.10$ \\
0.01 & $1.71 \pm 0.67 * *$ & $31.66 \pm 1.91^{* * *}$ & $1.35 \pm 0.15^{*}$ \\
0.1 & $0.92 \pm 0.49^{* * *}$ & $87.42 \pm 2.37 * * *$ & $3.44 \pm 0.35^{* *}$ \\
5 & $0.57 \pm 0.33^{* * *}$ & $145.82 \pm 9.94 * * *$ & $4.99 \pm 0.63 * * *$ \\
\hline
\end{tabular}

${ }^{*} p<0.05,{ }^{* *} p<0.01, * * * p<0.005, v s$. non-treated naïve mouse.

Table 2. The Mean Number of Anti-PEG IgM Molecules per SL

\begin{tabular}{cccc}
\hline \hline First dose $(\mathrm{nmol}$ PL/mouse $)$ & $N_{\mathrm{IgM} / \mathrm{SL}(\max )}($ molecule $)$ & $N_{\mathrm{IgM} / \mathrm{SL}}($ molecule $)$ & $N_{\mathrm{IgM} / \mathrm{SL}} / N_{\mathrm{IgM} / \mathrm{SL}(\max )}$ \\
\hline 0.01 & 8.34 & 1.51 & 0.181 \\
0.1 & 24.4 & 4.18 & 0.171 \\
5 & 62.3 & 9.21 & 0.148 \\
\hline
\end{tabular}

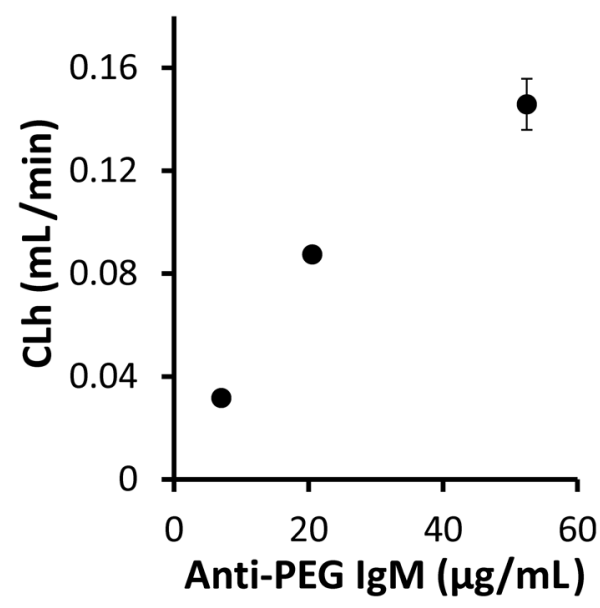

Fig. 4. Correlation between Serum Anti-PEG IgM Concentration and Hepatic Clearance of a Second Dose of SL

At day 5 after pretreatment with SL $(0.01,0.1$ or $5 \mathrm{nmol} \mathrm{PL} / \mathrm{mouse})$, mice were intravenously injected with radio-labeled SL ( $100 \mathrm{nmol} \mathrm{PL} / \mathrm{mouse})$. The relationship between $C L h$ and the concentration of anti-PEG IgM is shown. Data represent the mean \pm S.D. $(n=3)$.

CLs was increased, as the dose of the first injection was increased. As described above, the values were calculated by dividing the amount of SL accumulated in the liver and spleen, respectively, at $60 \mathrm{~min}$ post-injection by $A U C_{(0 \rightarrow 60 \mathrm{~min})}$. Thus, the CLh and CLs indicate which organ is the major one to clear the liposome under the ABC phenomenon. Under normal conditions (non-pretreated naïve mice), the value of CLh was 2.81-fold higher than the value of CLs. Under the ABC phenomenon (pretreated mice), the value of CLh became 25-fold higher than the value of $C L s$, indicating that hepatic clearance mainly contributed to the rapid clearance of SL from blood circulation.

The relationship between the $C L h$ and the serum anti-PEG IgM concentration was then evaluated (Fig. 4). The value of $C L h$ was increased and reached a plateau with increases in serum anti-PEG IgM concentration. This result indicates that the hepatic uptake of a second dose of SL may be a rate-limiting step in the $\mathrm{ABC}$ phenomenon.

The Number of Anti-PEG IgM per a SL Particle A second dose of SL was isolated from blood circulation following injection (100 nmol PL/mouse) after pretreatment with a first

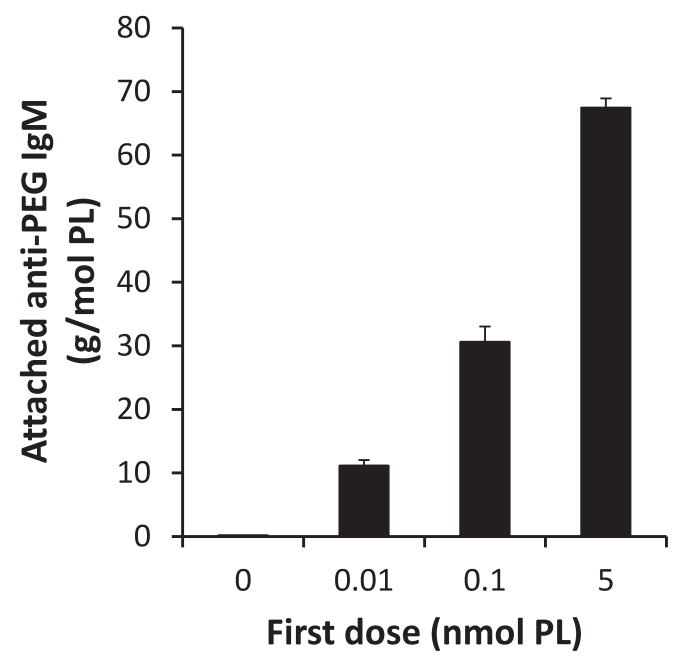

Fig. 5. The Amount of Associated Anti-PEG IgM on SL

At day 5 after pretreatment with SL $(0.01,0.1$ or $5 \mathrm{nmol} \mathrm{PL} / \mathrm{mouse})$, mice were intravenously injected with SL (100 nmol PL/mouse). Non-pretreated naïve mice were used as a control. Two minutes post-injection, the SL was isolated and lysed. The concentration of anti-PEG IgM in lysed SL solution was determined. Data represent the mean \pm S.D. $(n=3)$.

dose of SL (0.01, 0.1 or $5 \mathrm{nmol} \mathrm{PL} / \mathrm{mouse})$. Then, the amount of anti-PEG IgM associated with SL was quantitatively evaluated. As shown in Fig. 5, the amount of associated anti-PEG $\mathrm{IgM}$ on SL that could be isolated from mice that had received $5 \mathrm{nmol}$ PL as a first dose was $67.4 \mathrm{~g} / \mathrm{mol} \mathrm{PL}$. This amount was a 6.1- and 2.8-fold higher than that of mice receiving 0.01 and $0.1 \mathrm{nmol} \mathrm{PL} / \mathrm{mouse}$, respectively.

Then, to gain stoichiometric information, the number of anti-PEG IgM per one SL particle $\left(N_{\text {IgM/SL }}\right)$ was calculated. As shown in Table 2, the $N_{\text {IgM/SL }}$ in mice pretreated with 0.01 nmol PL showed only 1.51 molecules. Under these conditions, there was concern about the anti-PEG IgM free SL in blood circulation. This is supported by the results shown in Figs. 4A and B where $20 \%$ of a dose remained in circulation after intensive hepatic clearance in mice that had received $0.01 \mathrm{nmol} \mathrm{PL}$ as a first dose. The $N_{\mathrm{IgM} / \mathrm{SL}}$ in mice pretreated with 0.1 and $5 \mathrm{nmol}$ PL was sufficient to associate with all injected second-dose SL particles (4.18 and 9.21, respectively) and should completely remove the SL from the blood circulation. Only 15 to $17 \%$ of serum anti-PEG IgM was associated 
with a second dose of SL.

\section{DISCUSSION}

PEG is well known for its use with low immunogenic hydrophilic polymer, and modification of PEG onto nanoparticles extends the blood circulation of nanoparticles due to an ability to evade immune host survey system. However, various PEGylated nanocarriers, such as liposomes, ${ }^{10,12,27-29)}$ micelles, ${ }^{30)}$ emulsions ${ }^{12)}$ and solid lipid nanoparticles, ${ }^{13)}$ are known to induce anti-PEG IgM, which causes enhanced blood clearance of subsequent doses of PEGylated nanocarriers via opsonization by a complement system. In addition, such unexpected alterations of blood clearance have been observed in many animals such as mice, ${ }^{10)}$ rats $^{29,31)}$ rabbits $^{27)}$ and dogs. ${ }^{28)}$ The loss of the linear pharmacokinetics of PEGylated nanocarriers makes it difficult to predict the efficacy of encapsulated drugs in PEGylated nanoparticles upon repeated injection. Thus, control of the biodistribution of PEGylated nanocarriers is necessary in the $\mathrm{ABC}$ phenomenon. However, the relationship between an immune response against PEG and the immune clearance of PEGylated nanocarriers remains poorly understood.

In the present study, complement activation following a second injection of SL was increased linearly with increases in the serum anti-PEG $\operatorname{IgM}$ concentration irrespective of the $\mathrm{ABC}$ phenomenon (Fig. 2B). On the other hand, the CLh for a second dose, an indication of the intensity of the $\mathrm{ABC}$ phenomenon, might be increased sigmoidally, rather than linearly, with increases in the serum anti-PEG IgM concentration (Fig. 4). This observation indicates that complement activation and subsequent opsonization are not a rate-limiting process in the $\mathrm{ABC}$ phenomenon. In other words, the uptake process of Kupffer cells may be hampered by their limited capacity rather than by the preparation of opsonized SL. It is possible to envisage a two-phase model leading to the saturated hepatic uptake of a second dose of SL: first, there is the limited uptake capacity of Kupffer cells via complement receptor-mediated endocytosis/phagocytosis due to the number of available Kupffer cells and/or complement receptors on the Kupffer cells; second, there is the loss of interaction between complement receptors on Kupffer cells and complement-derived opsonins because of the rapid decomposition of complementderived opsonins on SL due to their unstable structures. ${ }^{32)}$ These factors could cause a sudden stop (saturation) of intensive hepatic uptake at/within $30 \mathrm{~min}$ (Fig. 3B).

Activation of the complement system by $\operatorname{IgMs}$ is known to rely on a conformational structure. ${ }^{33)}$ With an excess of antigen, the $\operatorname{IgM}$ attaches multivalently and forms a stable conformation. In an excess condition, however, the IgM attaches mono- or divalently and forms a starfish conformation. Since only a stable conformation of $\operatorname{IgM}$ can activate the complement system, an estimation of the anti-PEG IgM conformation on a SL is important. The SL used in this study had a surface area of about $34600 \mathrm{~nm}^{2}$ and about 3320 molecules of PEGylated lipids in an outer surface per each SL particle. The diameter and height of a stable IgM conformation is 25 and $10 \mathrm{~nm}$, respectively. ${ }^{34)} \mathrm{A}$ comparison of the respective areas showed that a theoretical maximum of about $55 \operatorname{IgM}$ molecules in a stable conformation would fit onto the surface of one SL unless one anti-PEG IgM occupied more than 60
$\mathrm{PEG}_{2000}$ molecules. Since this value was much higher than our calculated $N_{\mathrm{IgM} / \mathrm{SL}}$ (less than 10 molecules), it was likely that almost all of the anti-PEG IgMs bound on the SL had formed a stable conformation and all of the associated anti-PEG IgM had the potential to activate the complement system. Consistent with this estimation, linear relationships were observed between the intensity of the complement activation and the anti-PEG IgM concentration (Fig. 2B).

Although complement activation and rapid hepatic uptake appeared within $2 \mathrm{~min}$ (Figs. 2B, 3), it was unclear if all the anti-PEG IgM in the serum were associated with the SL following its post-injection. As shown in Table 2, the measured $N_{\text {IgM/SL }}$ was only $14-17 \%$ of what it had been 2 min postinjection. We reported that the anti-PEG IgM response against $\mathrm{SL}$ is a $\mathrm{T}$ cell-independent type 2 antibody response $\mathrm{e}^{35}$ ) and that one of the characteristics of IgM that is induced by a TI-2 antibody response is a low affinity against an immunized antigen. ${ }^{36)}$ Thus, our results may indicate that nearly $80 \%$ of the polyclonal anti-PEG IgM had a low affinity against PEG. This could lead to an assumption that only anti-PEG IgMs possessing a relatively high affinity can bind to SL rapidly and cause complement activation and rapid clearance. Unfortunately, our anti-PEG IgM-detecting ELISA system cannot distinguish differences in the affinities of anti-PEG IgMs in an antiserum. Further study is required to elucidate the affinities of antiPEG IgMs against PEG chains and PEGylated nanocarriers.

To date, with respect to the $\mathrm{ABC}$ phenomenon, researchers have mainly paid attention to the induction of anti-PEG $\operatorname{IgM}$ that is produced in response to a first dose of PEGylated nanoparticles. It is well known that not only induced $\mathrm{IgMs}$, but also naturally occurring IgMs against virus/bacteria protects against their infection and expansion. ${ }^{35)}$ It is noteworthy that naturally occurring anti-PEG IgMs have been observed in some patients and healthy subjects (about 4-25\%). ${ }^{37-39}$ ) Armstrong et al. have suggested that these naturally occurring anti-PEG antibodies could prime a host's immune response against treatment with PEGylated proteins, resulting in a reduced therapeutic effect. ${ }^{37)}$ There is concern that SL would interact with naturally occurring anti-PEG IgMs and trigger the $\mathrm{ABC}$ phenomenon. Our observations concerning the relationship between serum anti-PEG IgM concentration and indicators of the intensity of the accelerated blood clearance of SL may be useful in predicting the pharmacokinetic behavior of PEGylated nanocarriers upon repeated injections.

Acknowledgments The authors thank Mr. J. L. McDonald for his kind assistance in writing the manuscript. This study was supported, in part, by the Takeda Foundation and by a Grant-in-Aid for Scientific Research (B) (24390010) from the Ministry of Education, Culture, Sports, Science and Technology of Japan.

Conflict of Interest The authors declare no conflict of interest.

Supplementary Materials The online version of this article contains supplementary materials.

\section{REFERENCES}

1) Jokerst JV, Lobovkina T, Zare RN, Gambhir SS. Nanoparticle 
PEGylation for imaging and therapy. Nanomedicine, 6, 715-728 (2011).

2) Park K. Mechanistic study on the ABC phenomenon of PEG conjugates. J. Control. Release, 165, 234 (2013).

3) Allen TM, Hansen C. Pharmacokinetics of stealth versus conventional liposomes: effect of dose. Biochim. Biophys. Acta, 1068, 133-141 (1991).

4) Klibanov AL, Maruyama K, Torchilin VP, Huang L. Amphipathic polyethylene glycols effectively prolong the circulation time of liposomes. FEBS Lett., 268, 235-237 (1990).

5) Lasic DD, Martin FJ, Gabizon A, Huang SK, Papahadjopoulos D. Sterically stabilized liposomes: a hypothesis on the molecular origin of the extended circulation times. Biochim. Biophys. Acta, 1070, 187-192 (1991)

6) Allen C, Dos Santos N, Gallagher R, Chiu GNC, Shu Y, Li WM, Johnstone SA, Janoff AS, Mayer LD, Webb MS, Bally MB. Controlling the physical behavior and biological performance of liposome formulations through use of surface grafted poly(ethylene glycol). Biosci. Rep., 22, 225-250 (2002).

7) Torchilin VP, Omelyanenko VG, Papisov MI, Bogdanov AA Jr, Trubetskoy VS, Herron JH, Gentry CA. Poly(ethylene glycol) on the liposome surface: on the mechanism of polymer-coated liposome longevity. Biochim. Biophys. Acta, 1195, 11-20 (1994).

8) Maeda H. Macromolecular therapeutics in cancer treatment: the EPR effect and beyond. J. Control. Release, 164, 138-144 (2012).

9) Barenholz Y. Doxil ${ }^{\mathbb{R}}$ - the first FDA-approved nano-drug: lessons learned. J. Control. Release, 160, 117-134 (2012).

10) Abu Lila AS, Kiwada H, Ishida T. The accelerated blood clearance (ABC) phenomenon: clinical challenge and approaches to manage. J. Control. Release, 172, 38-47 (2013).

11) Dams ET, Laverman P, Oyen WJ, Storm G, Scherphof GL, van Der Meer JW, Corstens FH, Boerman OC. Accelerated blood clearance and altered biodistribution of repeated injections of sterically stabilized liposomes. J. Pharmacol. Exp. Ther., 292, 1071-1079 (2000).

12) Wang C, Cheng X, Sui Y, Luo X, Jiang G, Wang Y, Huang Z, She $Z$, Deng Y. A noticeable phenomenon: thiol terminal PEG enhances the immunogenicity of PEGylated emulsions injected intravenously or subcutaneously into rats. Eur. J. Pharm. Biopharm., 85, 744-751 (2013).

13) Zhao Y, Wang L, Yan M, Ma Y, Zang G, She Z, Deng Y. Repeated injection of PEGylated solid lipid nanoparticles induces accelerated blood clearance in mice and beagles. Int. J. Nanomedicine, 7, 2891-2900 (2012).

14) Szebeni J, Muggia F, Gabizon A, Barenholz Y. Activation of complement by therapeutic liposomes and other lipid excipient-based therapeutic products: prediction and prevention. Adv. Drug Deliv. Rev., 63, 1020-1030 (2011).

15) Ishida $T$, Harada $M$, Wang $X Y$, Ichihara $M$, Irimura $K$, Kiwada $\mathrm{H}$. Accelerated blood clearance of PEGylated liposomes following preceding liposome injection: effects of lipid dose and PEG surfacedensity and chain length of the first-dose liposomes. J. Control. Release, 105, 305-317 (2005).

16) Bartlett GR. Colorimetric assay methods for free and phosphorylated glyceric acids. J. Biol. Chem., 234, 469-471 (1959).

17) Shimizu T, Ichihara M, Yoshioka $Y$, Ishida $T$, Nakagawa S, Kiwada H. Intravenous administration of polyethylene glycol-coated (PEGylated) proteins and PEGylated adenovirus elicits an anti-PEG immunoglobulin M response. Biol. Pharm. Bull., 35, 1336-1342 (2012).

18) Hashimoto Y, Shimizu T, Mima Y, Abu Lila AS, Ishida T, Kiwada $\mathrm{H}$. Generation, characterization and in vivo biological activity of two distinct monoclonal anti-PEG IgMs. Toxicol. Appl. Pharmacol., 277, 30-38 (2014).

19) Ishihara T, Takeda M, Sakamoto M, Kimoto A, Kobayashi C, Takasaki N, Yuki K, Tanaka I, Takenaga M, Igarashi R, Maeda T, Ya- makawa N, Okamoto Y, Otsuka M, Ishida T, Kiwada H, Mizushima Y, Mizushima T. Accelerated blood clearance phenomenon upon repeated injection of PEG-modified PLA-nanoparticles. Pharm. Res., 26, 2270-2279 (2009).

20) Harashima H, Yamane C, Kume Y, Kiwada H. Kinetic analysis of $A U C$-dependent saturable clearance of liposomes: mathematical description of $A U C$ dependency. J. Pharmacokinet. Biopharm., 21, 299-308 (1993).

21) Takemoto S, Yamaoka K, Nishikawa M, Takakura Y. Histogram analysis of pharmacokinetic parameters by bootstrap resampling from one-point sampling data in animal experiments. Drug Metab. Pharmacokinet., 21, 458-464 (2006).

22) Wang X, Ishida T, Kiwada H. Anti-PEG IgM elicited by injection of liposomes is involved in the enhanced blood clearance of a subsequent dose of PEGylated liposomes. J. Control. Release, 119, 236-244 (2007).

23) Singh AK, Kilpatrick PK, Carbonell RG. Application of antibody and fluorophore-derivatized liposomes to heterogeneous immunoassays for $d$-dimer. Biotechnol. Prog., 12, 272-280 (1996).

24) Rus H, Cudrici C, Niculescu F. The role of the complement system in innate immunity. Immunol. Res., 33, 103-112 (2005).

25) Pham CTN, Mitchell LM, Huang JL, Lubniewski CM, Schall OF, Killgore JK, Pan DPJ, Wickline SA, Lanza GM, Hourcade DE. Variable antibody-dependent activation of complement by functionalized phospholipid nanoparticle surfaces. J. Biol. Chem., 286, 123-130 (2011).

26) Ishida T, Kiwada H. Accelerated blood clearance (ABC) phenomenon upon repeated injection of PEGylated liposomes. Int. J. Pharm., 354, 56-62 (2008).

27) Sroda K, Rydlewski J, Langner M, Kozubek A, Grzybek M, Sikorski AF. Repeated injections of PEG-PE liposomes generate antiPEG antibodies. Cell. Mol. Biol. Lett., 10, 37-47 (2005).

28) Suzuki T, Ichihara M, Hyodo K, Yamamoto E, Ishida T, Kiwada H, Ishihara H, Kikuchi H. Accelerated blood clearance of PEGylated liposomes containing doxorubicin upon repeated administration to dogs. Int. J. Pharm., 436, 636-643 (2012).

29) Li CL, Cao JN, Wang YJ, Zhao X, Deng CX, Wei N, Yang J, Cui JX. Accelerated blood clearance of pegylated liposomal topotecan: influence of polyethylene glycol grafting density and animal species. J. Pharm. Sci., 101, 3864-3876 (2012).

30) Kaminskas LM, Mcleod VM, Porter CJH, Boyd BJ. Differences in colloidal structure of PEGylated nanomaterials dictate the likelihood of accelerated blood clearance. J. Pharm. Sci., 100, 5069-5077 (2011).

31) Dams ETM, Laverman P, Oyen WJG, Storm G, Scherphof GL, Van der Meer JWM, Corstens FHM, Boerman OC. Accelerated blood clearance and altered biodistribution of repeated injections of sterically stabilized liposomes. J. Pharmacol. Exp. Ther., 292, 1071-1079 (2000).

32) Lynch DM, Kay PH, Papadimitriou JM, Grounds MD. Studies on the structure of complement $\mathrm{C} 3$ and the stability of $\mathrm{C} 3$ derived phagocytic ligands $\mathrm{C} 3 \mathrm{~b} / \mathrm{iC} 3 \mathrm{~b}$ in $\mathrm{SJL} / \mathrm{J}$ and $\mathrm{BALB} / \mathrm{c}$ mice. Eur. J. Immunogenet., 20, 1-9 (1993).

33) Borsos T, Chapuis RM, Langone JJ. Distinction between fixation of $\mathrm{C} 1$ and the activation of complement by natural IgM anti-hapten antibody: effect of cell surface hapten density. Mol. Immunol., 18, 863-868 (1981).

34) Armstrong SJ, Outlaw MC, Dimmock NJ. Morphological studies of the neutralization of influenza virus by IgM. J. Gen. Virol., 71, 2313-2319 (1990).

35) Ochsenbein AF, Zinkernagel RM. Natural antibodies and complement link innate and acquired immunity. Immunol. Today, 21, $624-630(2000)$.

36) Jones HE, Taylor PR, McGreal E, Zamze S, Wong SYC. The contribution of naturally occurring IgM antibodies, IgM cross-reactivity and complement dependency in murine humoral responses to 
pneumococcal capsular polysaccharides. Vaccine, 27, 5806-5815 (2009).

37) Armstrong JK, Hempel G, Koling S, Chan LS, Fisher T, Meiselman HJ, Garratty G. Antibody against poly(ethylene glycol) adversely affects PEG-asparaginase therapy in acute lymphoblastic leukemia patients. Cancer, 110, 103-111 (2007).

38) Liu Y, Reidler H, Pan J, Milunic D, Qin D, Chen D, Vallejo YR,
Yin R. A double antigen bridging immunogenicity ELISA for the detection of antibodies to polyethylene glycol polymers. J. Pharmacol. Toxicol. Methods, 64, 238-245 (2011).

39) Richter AW, Akerblom E. Antibodies against polyethylene glycol produced in animals by immunization with monomethoxy polyethylene glycol modified proteins. Int. Arch. Allergy Appl. Immunol., 70, 124-131 (1983). 